\title{
A 4-item PRECISE-DAPT score for dual antiplatelet therapy duration decision-making
}

Francesco Costa, MD, ${ }^{\mathrm{a}}$ David van Klaveren, $\mathrm{PhD},{ }^{\mathrm{b}}$ Antonio Colombo, $\mathrm{MD},{ }^{\mathrm{c}}$ Fausto Feres, MD, ${ }^{\mathrm{d}}$ Lorenz Räber, $\mathrm{MD},{ }^{\mathrm{e}}$ Thomas Pilgrim, MD, ${ }^{\mathrm{e}}$ Myeong-Ki Hong, MD, ${ }^{\mathrm{f}, \mathrm{g}}$ Hyo-Soo Kim, MD, ${ }^{\mathrm{h}}$ Stephan Windecker, MD, ${ }^{\mathrm{e}}$ Ewout W. Steyerberg, PhD, ${ }^{b}$ and Marco Valgimigli, MD $^{\mathrm{e}}$, , for the PRECISE-DAPT Study Investigators Cotignola, Italy; Sao Paulo, Brazil; and Seoul, Republic of Korea

\begin{abstract}
The originally-proposed PRECISE-DAPT score is a 5-item risk score supporting decision-making for dual antiplatelet therapy ${ }^{1}$ duration after $\mathrm{PCl}$. It is unknown if a simplified version of the score based on 4 factors (age, hemoglobin, creatinine clearance, prior bleeding), and lacking white-blood cell count, retains potential to guide DAPT duration. The 4-item PRECISEDAPT was used to categorize 10,081 patients who were randomized to short (3-6 months) or long (12-24 months) DAPT regimen according to high (HBR defined by PRECISE-DAPT $\geq 25$ points) or non-high bleeding risk (PRECISE-DAPT $<25$ ) status. Long treatment duration was associated with higher bleeding rates in HBR (ARD $+2.22 \%[95 \% \mathrm{Cl}+0.53$ to +3.90$]$ ) but not in non-HBR patients (ARD $+0.25 \%[-0.14$ to +0.64$] ; p_{\text {int }}=0.026$ ), and associated with lower ischemic risks in non-HBR (ARD $-1.44 \%[95 \% \mathrm{Cl}-2.56$ to -0.31$])$, but not in HBR patients (ARD $+1.16 \%[-1.91$ to +4.22$] ; p_{\text {int }}=0.11$ ). Only non-HBR patients experienced lower net clinical adverse events (NACE) with longer DAPT ( $p_{\text {int }}=0.043$ ). A 4-item simplified version of the PRECISE-DAPT score retains the potential to categorize patients who benefit from prolonged DAPT without concomitant bleeding liability from those who do not. (Am Heart J 2020;223:44-47.)
\end{abstract}

\section{Introduction}

The PRECISE-DAPT score is a 5-item bleeding risk prediction tool developed for patients treated with dual antiplatelet therapy. ${ }^{1,2}$ By stratifying patients according to the baseline bleeding risk, PRECISE-DAPT has shown potential to inform decision-making for DAPT duration after percutaneous coronary intervention ${ }^{1-3}$ and it has been endorsed by international guidelines and routine clinical practice worldwide. ${ }^{4} \mathrm{~A}$ simplified version of this score lacking white blood cells count (WBC) and therefore based on 4 factors (i.e. age, hemoglobin, creatinine clearance, prior bleeding requiring medical attention) has been previously generated, showing only minimal discrimination or calibration losses as compared $\mathrm{t} \mathrm{O}$

th-From the "Department of Clinical and Experimental Medicine, Policlinic "G. Martino", e University of Messina, Italy, 'b Erasmus University Medical Center, s-Gravendijkwal 230, Rotterdam, The Netherlands, Interventional Cardiology Unit, GVM Care \& 5 -Research Maria Cecilia Hospital, Cotignola, Italy, dIstituto Dante Pazzanese de Cardiologia, Sao Paulo, Brazil, 'Swiss Cardiovascular Center Bern, Bern University

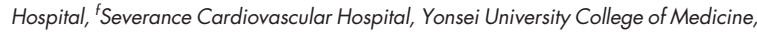
Seoul, Republic of Korea, ${ }^{9}$ Severance Biomedical Science Institute, Yonsei University College of Medicine, Seoul, Republic of Korea, and ${ }^{h}$ Department of Internal Medicine, Seoul National University Hospital, Seoul, Republic of Korea.

${ }^{2}$ Funding: No additional external funding was usedDisclosures: The authors are solely responsible for the design and conduct of this study, all study analyses, the drafting and editing of the manuscript, and its final contents.

Submitted October 30, 2019; accepted January 24, 2020.

Reprint requests: Marco Valgimigli MD PhD FESC, Swiss Cardiovascular Center Bern

University of Bern, CH-3010, Bern, Switzerland.

E-mail: marco.valgimigli@insel.ch

$0002-8703$

(C) 2020 Published by Elsevier Inc.

https://doi.org/10.1016/i.ahj.2020.01.014 item iteration in both the generation and validation datasets. $^{2}$ The aim of the current analysis was to test whether this simplified version of the score helps categorizing patients at high bleeding risk (HBR) who should receive shortened DAPT after PCI.

\section{Methods}

We sought to assess the role of a 4 -item PRECISE-DAPT score to guide decision-making on DAPT duration across 10,081 patients who were randomly allocated to short (3 or 6 months) or long (12 or up to 24 months) DAPT duration within the PRECISE DAPT pooled dataset. Details regarding the individual study inclusion/exclusion criteria, procedural characteristics, endpoints definitions event adjudication and regulatory approval were previously reported. ${ }^{2}$ The 4 -item simplified PRECISE-DAPT was obtained by excluding white blood cell count (WBC) at baseline, and by re-weighing the other 4 score components within the multivariable model as previously described. $^{2}$ Score discrimination and calibration were previously evaluated in both the generation cohort, and in two, independent, validation cohorts. ${ }^{2}$

In the current analysis, we assessed the impact of the randomly allocated short and long DAPT durations on bleeding (i.e. TIMI major or minor definition), ischemic events (i.e. composite of myocardial infarction, stent thrombosis, stroke or target vessel revascularization), and net adverse clinical events (NACE, i.e. the combination of aforementioned ischemic and bleeding events) across the 


\section{Figure 1}

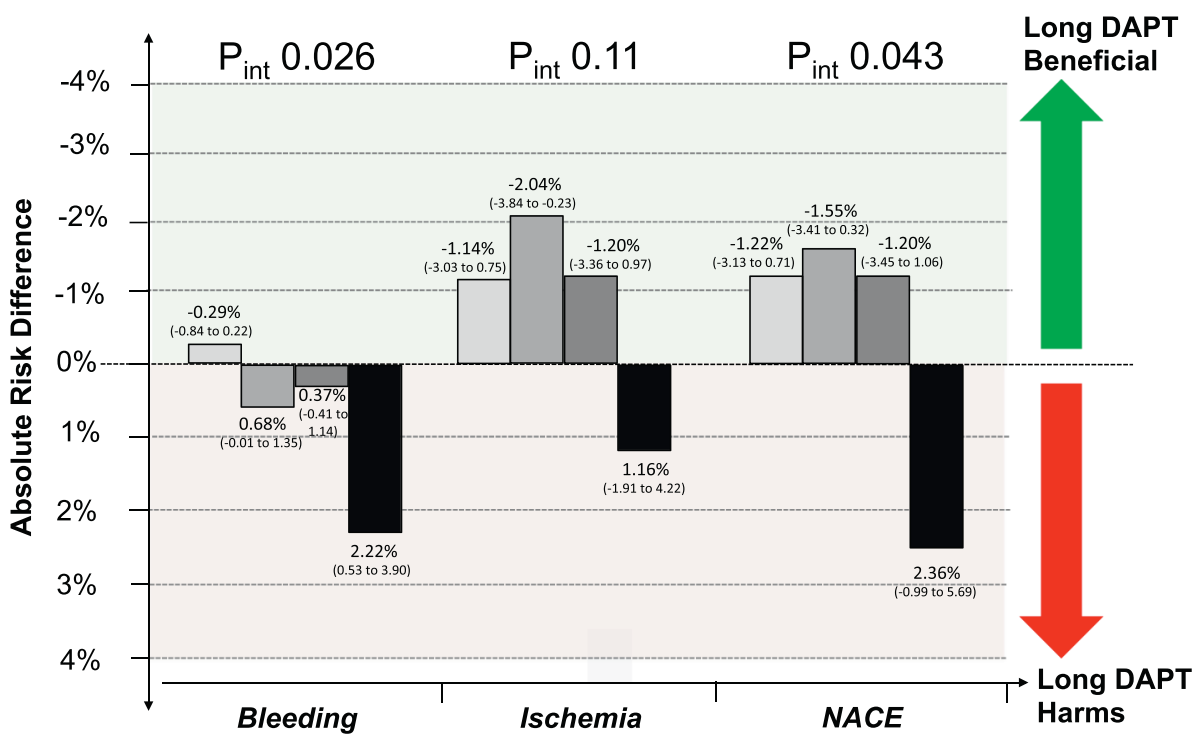

PRECISE-DAPT
Simplified
Score
Non-High score:
High score
$\leq 7$ points (Very-Low)
8-15 points (Low)
16-24 points (Moderate)
$\geq 25$ points (High)

Impact of dual antiplatelet therapy duration across the 4-item PRECISE-DAPT score quartiles. Absolute risk difference (ARD) for long (12-24 months) as compared to short (3-6 months) DAPT duration across the 4-item PRECISE-DAPT score quartiles for bleeding (TIMI major/minor definition), ischemic (composite of myocardial infarction, definite stent thrombosis, stroke or target vessel revascularization) and net adverse clinical events (NACE) (composite of ischemic and bleeding endpoints) are presented. Bars plotted on the upper side of the zero line represent benefit of long versus short DAPT duration, whereas bars plotted on the lower side of the zero line represent harm from long versus short DAPT duration.

simplified 4-item PRECISE-DAPT score quartiles (i.e. verylow $\leq 7$ points, low 8 to 15 points, moderate 16 to 24 points, high $\geq 25$ points). Interaction between high (highest quartile) versus non-high (lowest three quartiles) 4-item PRECISE DAPT score and DAPT duration was evaluated by assessing heterogeneity for absolute risk differences. All analysis were performed with $\mathrm{R}$ version 3.6 (R Foundation, Vienna, Austria), and a $P<.05$ was considered for statistical significance. No extramural funding was used to support this work.

\section{Results}

The median simplified 4-item PRECISE-DAPT score was 14.8 points (IQR: $7-24$ ) and a total of 3496 patients (23.4\%) were considered at $\mathrm{HBR}$ (ie, score $\geq 25$ points). Longer treatment duration was associated with a significant excess of bleeding events exclusively in HBR patients (ARD $+2.22 \%$ [95\% CI +0.53 to +3.90]; number needed to treat [NNT]: 45) but not in those without HBR profile (i.e., very low, low or moderate risk: $\mathrm{ARD}+0.25 \%[-0.14$ to +0.64$\left.] ; \mathrm{p}_{\text {int }}=0.026\right)($ Figure 1). This remained consistent when only events occurring during the first year after PCI were accounted for $\left(p_{\text {int }}=0.042\right)$. Concurrently, longer DAPT duration was associated with lower rates of the composite ischemic endpoint of myocardial infarction, definite stent thrombosis, stroke, or target vessel revascularization in non-HBR patients (ARD -1.44\% [95\% CI -2.56 to -0.31]; NNT: 69), but not in those at HBR $\left(\mathrm{ARD}+1.16 \%[-1.91\right.$ to +4.22$\left.] ; \mathrm{p}_{\text {int }}=0.11\right)($ Figure 1). When both ischemic and bleeding endpoints were simultaneously considered in a NACE endpoint, non-HBR patients benefitted from longer DAPT duration (ARD -1.29\% [95\% CI -2.46 to -0.13]; NNT: 77), while HBR patients did not (ARD $+2.36 \%$ [95\% CI -0.99 to 5.69]; $\left.\mathrm{p}_{\mathrm{int}}=0.043\right)($ Figure 1).

\section{Discussion}

In the current analysis, which was carried out in a pooled dataset of 5 randomized studies and including more than 10,000 patients, we show for the first time that a simplified 4-item PRECISE-DAPT score (i.e. excluding WBC) may prove useful to support clinical decisionmaking for DAPT duration. At a cut-off of 25 points 
(consistent with that provided by the 5-item score), the simplified PRECISE-DAPT score was able to identify patients at higher bleeding risk who do not apparently derive benefits, and may actually be harmed, by longer treatment duration. On the other hand, non-HBR patients derived ischemic benefit from longer DAPT duration without being exposed to significant bleeding risk. Hence, the simplified 4-item PRECISE-DAPT score retains the ability to inform DAPT duration decision-making. Personalized DAPT treatment after stenting has been extensively investigated in recent years, ${ }^{4-7}$ and several clinical and technical characteristics, ${ }^{8-10}$ as well as clinical risk scores, ${ }^{11}$ were shown to support clinical decision making. ${ }^{4,6}$ The PRECISE-DAPT score implements bleeding risk status at the time of PCI and it was previously shown to provide decision-making potentials for short versus long-term DAPT duration even in patients at concomitantly very high ischemic risk such as those with ACS undergoing complex PCI. ${ }^{1,2}$ Yet, despite this tool has been widely endorsed from international societies and guidelines, and widely implemented in real world practice, several barriers for the routine application of risk scores still exist. ${ }^{12}$

On this matter, the simplified 4-item iteration of the PRECISE-DAPT score presented in the current analysis may prove useful for at least three reasons: first, accounting on 4 , rather than 5 , factors, allows for faster score computation in clinical practice; second, WBC levels change over time during hospital stay, especially in acute coronary syndrome patients, and may create ambiguity in patient categorization. ${ }^{13}$ In this case, a second evaluation with this simplified tool may facilitate a more unbiased risk assessment. Third, this version of the score may facilitate prospective or retrospective computation of the PRECISE-DAPT score in various datasets in which WBC values is not available.

\section{Conclusions}

A simplified 4-item PRECISE-DAPT score excluding WBC maintains the capability to categorize patients who benefit or not from prolonged DAPT, and may offer an alternative solution for risk stratification and decision making purposes in settings where WBC is not available.

Am Heart J 2020;44-47.

$0002-8703$

https://doi.org/10.1016/i.ahj.2020.01.014

Francesco Costa, MD

David van Klaveren, $P b D$

Antonio Colombo, MD

Fausto Feres, MD

Lorenz Rðber, MD

Thomas Pilgrim, MD

Myeong-Ki Hong, MD
Hyo-Soo Kim, MD

Stephan Windecker, MD

Ewout W. Steyerberg, PbD

Marco Valgimigli, MD

Department of Clinical and Experimental Medicine

Policlinic "G. Martino"

University of Messina

Italy

Erasmus University Medical Center

s-Gravendijkwal 230

Rotterdam

The Netherlands

Interventional Cardiology Unit

GVM Care \& Research Maria Cecilia Hospital

Cotignola

Italy

Istituto Dante Pazzanese de Cardiologia

Sao Paulo

Brazil

Swiss Cardiovascular Center Bern

Bern University Hospital

Severance Cardiovascular Hospital

Yonsei University College of Medicine

Seoul

Republic of Korea

Severance Biomedical Science Institute

Yonsei University College of Medicine

Seoul

Republic of Korea

Department of Internal Medicine

Seoul National University Hospital

Seoul, Republic of Korea

E-mail: marco.valgimigli@insel.ch

\section{References}

1. Costa F, Van Klaveren D, Feres F, et al. Dual Antiplatelet Therapy Duration Based on Ischemic and Bleeding Risks After Coronary Stenting. J Am Coll Cardiol 2019;73(7):741-54.

2. Costa F, van Klaveren $D$, James $S$, et al. Derivation and validation of the predicting bleeding complications in patients undergoing stent implantation and subsequent dual antiplatelet therapy (PRECISEDAPT) score: a pooled analysis of individual-patient datasets from clinical trials. Lancet 2017;389.

3. Cannon CP, Gropper S, Bhatt DL, et al. Design and Rationale of the RE-DUAL PCI Trial: a prospective, randomized, phase $3 b$ study comparing the safety and efficacy of dual antithrombotic therapy with dabigatran etexilate versus warfarin triple therapy in patients with nonvalvular atrial fibrillation who have undergone percutaneous coronary intervention with stenting. Clin Cardiol 2016;39(10): 555-64.

4. Valgimigli $M$, Bueno $H$, Byrne RA, et al. ESC focused update on dual antiplatelet therapy in coronary artery disease developed in collaboration with EACTS: The Task Force for dual antiplatelet therapy in coronary artery disease of the European Society of Cardiology (ESC) and of the European Association for Cardio-Thoracic Surgery (EACTS). Eur Heart J 2017:2017 
5. Costa F, Adamo M, Ariotti S, et al. Impact of greater than 12-month dual antiplatelet therapy duration on mortality: Drug-specific or a class-effect? A meta-analysis. Int J Cardiol 2015;201:179-81.

6. Valgimigli $M$, Costa F, Byrne R, et al. Dual antiplatelet therapy duration after coronary stenting in clinical practice: results of an EAPCI survey. Eurolntervention 2015;1 1(1):68-74.

7. Costa F, Windecker S, Valgimigli M. Dual antiplatelet therapy duration: reconciling the inconsistencies. Drugs 2017;77(16): 1733-54.

8. Costa F, Vranckx P, Leonardi S, et al. Impact of clinical presentation on ischaemic and bleeding outcomes in patients receiving 6- or 24month duration of dual-antiplatelet therapy after stent implantation: a pre-specified analysis from the PRODIGY (Prolonging DualAntiplatelet Treatment After Grading Stent-Induced Intimal Hyperplasia) trial. Eur Heart J 2015;36(20):1242-51.

9. Costa F, Adamo M, Ariotti S, et al. Left main or proximal left anterior descending coronary artery disease location identifies high-risk patients deriving potentially greater benefit from prolonged dual antiplatelet therapy duration. Eurolntervention 2016;11(11): e1222-30.

10. Crimi G, Leonardi S, Costa F, et al. Role of stent type and of duration of dual antiplatelet therapy in patients with chronic kidney disease undergoing percutaneous coronary interventions. Is bare metal stent implantation still a justifiable choice? A post-hoc analysis of the all comer PRODIGY trial. Int J Cardiol 2016;212:1 10-7.

11. Costa F, Tijssen JG, Ariotti S, et al. Incremental value of the CRUSADE, ACUITY, and HAS-BLED risk scores for the prediction of hemorrhagic events after coronary stent implantation in patients undergoing long or short duration of dual antiplatelet therapy. J Am Heart Assoc 2015;4(12), e002524.

12. Eichler K, Zoller M, Tschudi P, et al. Barriers to apply cardiovascular prediction rules in primary care: a postal survey. BMC Fam Pract 2007;8:1.

13. Shah $A D$, Denaxas $S$, Nicholas $O$, et al. Initial presentation of 12 cardiovascular diseases: a CALIBER Cohort Study. J Am Coll Cardiol 2017;69(9):1160-9. 